PROCEEDINGS OF THE

AMERICAN MATHEMATICAL SOCIETY

Volume 138, Number 4, April 2010, Pages 1263-1272

S 0002-9939(09)10188-0

Article electronically published on December 8, 2009

\title{
PARTITION VALUES AND CENTRAL CRITICAL VALUES OF CERTAIN MODULAR $L$-FUNCTIONS
}

\author{
JOHN J. WEBB
}

(Communicated by Ken Ono)

\begin{abstract}
Let $p(n)$ denote the number of partitions of a positive number $n$, let $\ell \in\{5,7,11\}$ and let $\delta_{\ell}$ be the least non-negative residue of $24^{-1}$ modulo $\ell$. In this paper we prove congruences modulo $\ell$ between $\frac{p\left(\ell n+\delta_{\ell}\right)}{\ell}$ and ratios of central critical values of $L$-functions associated to twists of certain integer weight newforms. In 1999, Guo and Ono proved analogous results for $13 \leq$ $\ell \leq 31$.
\end{abstract}

\section{INTRODUCTION}

Let $p(n)$ be the number of partitions of a positive integer $n$ : the number of non-increasing sequences of positive integers whose sum is $n$. By convention, we agree that $p(0):=1$. Partitions play an important role in number theory, combinatorics, representation theory, and Lie algebras. See, for example, And98, Ono04, Ono08 and the references therein. The infinite product generating function of Euler,

$$
\sum_{n=0}^{\infty} p(n) q^{n}=\prod_{m=1}^{\infty}\left(1-q^{m}\right)^{-1}
$$

is the starting point for much of the modern study of $p(n)$. Using this formula, Ramanujan discovered and often proved interesting results on the arithmetic of $p(n)$. The most famous of these are the Ramanujan congruences, which we now describe. Let $\ell \geq 5$ be prime, and define

$$
\delta_{\ell}:=\ell\left(1+\left\lfloor\frac{\ell}{24}\right\rfloor\right)-\left(\frac{\ell^{2}-1}{24}\right) .
$$

Note that $\delta_{\ell}$ is the least non-negative residue of $24^{-1}$ modulo $\ell$. Ramanujan proved that if $\ell \in\{5,7,11\}$, then for all $n \geq 0$ we have

$$
p\left(\ell n+\delta_{\ell}\right) \equiv 0 \quad(\bmod \ell) .
$$

These congruences have inspired terrific interest in the general study of linear congruences for Fourier coefficients of modular forms. Using the fact that (1.1) is closely related to a weakly holomorphic modular form of weight $-1 / 2$, Ahlgren and Ono in Ahl00, AO01, and Ono00 proved that congruences for $p(n)$ are actually common in the following sense: for all $m \geq 1$ with $\operatorname{gcd}(m, 6)=1$, there are

Received by the editors August 28, 2009.

2010 Mathematics Subject Classification. Primary 11F33; Secondary 11F11.

(C)2009 American Mathematical Society 
infinitely many non-nested arithmetic progressions $A n+B$ such that $p(A n+B) \equiv 0$ $(\bmod m)$ for all non-negative integers $n$. On the other hand, Ahlgren and Boylan AB03 proved that congruences of the type that Ramanujan found are quite rare; to be precise, the only $(\ell, d)$ with $\ell$ prime such that $p(\ell n+d) \equiv 0(\bmod \ell)$ are $(5,4)$, $(7,5)$ and $(11,6)$.

1.1. Statement of results. In this paper we connect values of $p\left(\ell n+\delta_{\ell}\right)$ for $\ell \in\{5,7,11\}$ to central critical values of twists of modular $L$-functions. We begin by fixing some notation. Let $\ell \geq 5$ be prime. We define integers $\lambda_{\ell}$ and $r_{\ell}$ by

$$
\begin{gathered}
\lambda_{\ell}:= \begin{cases}9 & \ell=5, \\
8 & \ell=7, \\
14 & \ell=11, \\
\frac{\ell-3}{2} & \ell \geq 13,\end{cases} \\
r_{\ell}:=24\left\lceil\frac{\ell}{24}\right\rceil-\ell .
\end{gathered}
$$

Observe that $r_{\ell}$ is the least non-negative residue of $-\ell$ modulo 24 . For non-negative integers $n$, define $D(\ell, n)$ by

$$
D(\ell, n):=(-1)^{\lambda_{\ell}}\left(24 n+r_{\ell}\right) .
$$

Suppose that $D(\ell, n)$ is square-free. Then it is a fundamental discriminant (i.e., the discriminant of a quadratic number field). If $t$ is a square-free integer, we denote by $\chi_{t}(\cdot)$ the Kronecker character associated to $\mathbb{Q}(\sqrt{t})$. In particular, we have $\chi_{D(\ell, n)}(\cdot)=\left(\frac{D(\ell, n)}{\cdot}\right)$. We define $\chi_{12}$ to be the character modulo 12 induced from the Kronecker character associated to $\mathbb{Q}(\sqrt{3})$.

For primes $\ell \geq 3$, let $(\dot{\bar{\ell}})$ denote the Legendre symbol modulo $\ell$. When $5 \leq \ell \leq$ 31 , one may verify that there exists a unique newform $G_{\ell}(z)=\sum_{m=1}^{\infty} a_{\ell}(m) q^{m} \in$ $S_{2 \lambda_{\ell}}\left(\Gamma_{0}(6)\right)$ whose $q$-expansion is

$$
G_{\ell}(z):=q+\left(\frac{2}{\ell}\right) 2^{\lambda_{\ell}-1} q^{2}+\left(\frac{3}{\ell}\right) 3^{\lambda_{\ell}-1} q^{3}+\ldots
$$

Our result concerns the central critical values of the $L$-functions associated to $G_{\ell}(z)$ twisted by the Kronecker characters $\chi_{D(\ell, n)}$. For $s \in \mathbb{C}$ with $\Re(s) \geq \lambda_{\ell}+\frac{1}{2}$ and $D(\ell, n)$ square-free we define the associated $L$-function for $G_{\ell}(z)$ twisted by $\chi_{D(\ell, n)}$ in the usual way as

$$
L\left(G_{\ell} \otimes \chi_{D(\ell, n)}, s\right):=\sum_{m=1}^{\infty} \chi_{D(\ell, n)}(m) a_{\ell}(m) m^{-s} .
$$

This function has an analytic continuation to all of $\mathbb{C}$ and satisfies a functional equation centered at the line $s=\lambda_{\ell}$. We now state our main result.

Theorem 1.1. Let $\ell \in\{5,7,11\}$, let $n \geq 0$ be an integer and let $\delta_{\ell}, \lambda_{\ell}, r_{\ell}, D(\ell, n)$, and $G_{\ell}(z)$ be defined as in (1.2), (1.4), (1.5), (1.6), and (1.7). Then if $D(\ell, n)$ is square-free, we have

$$
\frac{L\left(G_{\ell} \otimes \chi_{D(\ell, n)}, \lambda_{\ell}\right)\left(24 n+r_{\ell}\right)^{\lambda_{\ell}-\frac{1}{2}}}{L\left(G_{\ell} \otimes \chi_{D(\ell, 0)}, \lambda_{\ell}\right) r_{\ell}^{\lambda_{\ell}-\frac{1}{2}}} \equiv \frac{p\left(\ell n+\delta_{\ell}\right)^{2}}{\ell^{2}} \quad(\bmod \ell) .
$$


The method of proof entails finding a generating function for $\frac{p\left(\ell n+\delta_{\ell}\right)}{\ell}$ modulo $\ell$ that is a half-integral weight modular form to which we apply powerful theorems of Shimura and Waldspurger. The relevant modular forms lie in vector spaces of large dimension, which makes the necessary computations to confirm our results non-trivial.

Remark 1.2. Guo and Ono in GO99 proved that Theorem 1.1 holds for primes $13 \leq \ell \leq 31$ (replacing $\frac{p\left(\ell n+\delta_{\ell}\right)^{2}}{\ell^{2}}$ with $\frac{p\left(\ell n+\delta_{\ell}\right)^{2}}{p\left(\delta_{\ell}\right)^{2}}$ in the statement). To prove our result, we extend their methods to account for the Ramanujan congruences.

Remark 1.3. In [GO99], Guo and Ono use their result for $13 \leq \ell \leq 31$ to show that the Bloch-Kato Conjecture implies that there is a close relationship between the $\ell$-divisibility of $p\left(\ell n+\delta_{\ell}\right)$ and the $\ell$-divisibility of orders of the Tate-Shafarevich groups of twists of motives associated to $G_{\ell}$. They proved, assuming the Bloch-Kato conjecture, that $\ell$ divides the order of the relevant Tate-Shafarevich group if and only if $p\left(\ell n+\delta_{\ell}\right) \equiv 0(\bmod \ell)$. For $\ell \in\{5,7,11\}$, an analogous argument assuming the Bloch-Kato Conjecture and using Theorem 1.1 would imply $\ell$-divisibility results for orders of associated Tate-Shafarevich groups when $p\left(\ell n+\delta_{\ell}\right) \equiv 0\left(\bmod \ell^{2}\right)$.

Remark 1.4. One may verify results of this type for a fixed prime $\ell$ using our methods and those of Guo and Ono via a finite computation. Therefore, our ability to verify analogous results for fixed primes $\ell>31$ only depends on our ability to do large computations. Moreover, since our method entails separate computations for each prime, we are unable to apply it to prove a uniform result for all primes $\ell \geq 5$.

For primes $\ell \in\{5,7,11\}$, we have $\frac{p\left(\ell n+\delta_{\ell}\right)^{2}}{\ell^{2}} \not \equiv 0(\bmod \ell)$ if and only if $p\left(\ell n+\delta_{\ell}\right) \not \equiv$ $0\left(\bmod \ell^{2}\right)$. Therefore we obtain the following result on the non-vanishing of central critical values of the $L$-functions of the type occurring in Theorem 1.1 .

Corollary 1.5. Let $n \geq 0$ be an integer for which $D(\ell, n)$ is square-free and suppose that $p\left(\ell n+\delta_{\ell}\right) \not \equiv 0\left(\bmod \ell^{2}\right)$. Then we have

$$
L\left(G_{\ell} \otimes \chi_{D(\ell, n)}, \lambda_{\ell}\right) \neq 0 .
$$

To illustrate the corollary, we study the function

$$
R_{\ell}(X):=\frac{\#\left\{0 \leq n \leq X: p\left(\ell n+\delta_{\ell}\right) \not \equiv 0\left(\bmod \ell^{2}\right), D(\ell, n) \text { square-free }\right\}}{\#\{0 \leq n \leq X: D(\ell, n) \text { square-free }\}}
$$

This function gives the proportion of non-negative integers $n \leq X$ such that Corollary 1.5 implies the non-vanishing of $L\left(G_{\ell} \otimes \chi_{D(\ell, n)}, \lambda_{\ell}\right)$. Our calculations reveal the following data:

\begin{tabular}{|c|c|c|c|}
\hline$X$ & $R_{5}(X)$ & $R_{7}(X)$ & $R_{11}(X)$ \\
\hline 1000 & 0.680829 & 0.364629 & 0.802186 \\
10000 & 0.662284 & 0.371357 & 0.834448 \\
100000 & 0.664233 & 0.371774 & 0.849149 \\
900000 & 0.662061 & 0.372700 & 0.857340 \\
\hline
\end{tabular}

The data is heavily influenced by extensions of (1.3) to modulus $\ell^{2}$ (see for example Kno93]) given by

$$
\begin{array}{rlll}
p(25 n+24) & \equiv & 0 & (\bmod 25), \\
p(49 n+d) & \equiv & 0 & (\bmod 49) \\
p(121 n+116) & \equiv & 0 & (\bmod 121)] .
\end{array} \quad \text { for } d \in\{19,33,40,47\}
$$


For $\ell \in\{5,7,11\}$ we let $\mathcal{M}_{\ell}$ be the set of non-negative integers $n$ such that $\ell n+\delta_{\ell}$ falls into the progressions in (1.9); i.e., we let

$$
\mathcal{M}_{\ell}:= \begin{cases}\{n \geq 0: n \equiv 4 \quad(\bmod 5)\} & \ell=5, \\ \{n \geq 0: n \equiv 2,4,5,6 \quad(\bmod 7)\} & \ell=7, \\ \{n \geq 0: n \equiv 10 \quad(\bmod 11)\} & \ell=11 .\end{cases}
$$

Hence, we may renormalize $R_{\ell}(X)$ to account for the congruences (1.9) by defining $R_{\ell}^{\prime}(X):=\frac{\#\left\{0 \leq n \leq X: p\left(\ell n+\delta_{\ell}\right) \not \equiv 0\left(\bmod \ell^{2}\right), D(\ell, n) \text { square-free, } n \notin \mathcal{M}_{\ell}\right\}}{\#\left\{0 \leq n \leq X: D(\ell, n) \text { square-free, } n \notin \mathcal{M}_{\ell}\right\}}$.

The data for $R_{\ell}^{\prime}(X)$ is

\begin{tabular}{|c|c|c|c|}
\hline$X$ & $R_{5}^{\prime}(X)$ & $R_{7}^{\prime}(X)$ & $R_{11}^{\prime}(X)$ \\
\hline 1000 & 0.819135 & 0.847716 & 0.838517 \\
10000 & 0.795368 & 0.851721 & 0.871350 \\
100000 & 0.797205 & 0.850389 & 0.886614 \\
900000 & 0.794490 & 0.852020 & 0.894960 \\
\hline
\end{tabular}

Remark 1.6. We note that for primes $13 \leq \ell \leq 31$, the data in G099] show that $R_{\ell}(X)$ as in (1.8) seems to be slightly less than $1-\frac{1}{\ell}$ as $X$ gets large. Our data show that for primes $\ell \in\{5,7,11\}$, the normalization of $R_{\ell}(X)$ given by $R_{\ell}^{\prime}(X)$ exhibits similar behavior as $X$ gets large.

The paper is structured as follows. In Section 2 we introduce the necessary facts from the theory of modular forms that will be used in our proof. Section 3 contains the proof of our theorem. We include an appendix to discuss some of the details of our computations.

\section{FACTS ON MODULAR FORMS}

In this section we give the necessary facts on modular forms that we require. For details on the theory, one may consult for example Iwa97 or Ono04.

Let $\mathfrak{h}$ be the complex upper half-plane, and let $q:=e^{2 \pi i z}$. For integers $k \geq 0$ and $N \geq 1$, and $\chi$, a Dirichlet character modulo $N$, we let $M_{k}\left(\Gamma_{0}(N), \chi\right)$ denote the $\mathbb{C}$-vector space of weight $k$ holomorphic modular forms on $\Gamma_{0}(N)$ with character $\chi$. We denote by $S_{k}\left(\Gamma_{0}(N), \chi\right)$ the subspace of cusp forms. When $\chi$ is trivial, we often omit it.

For even $k \geq 4$, let $B_{k}$ be the $k$-th Bernoulli number and define the Eisenstein series $E_{k}$ by

$$
E_{k}(z):=1-\frac{2 k}{B_{k}} \sum_{n=1}^{\infty} \sum_{d \mid n} d^{k-1} q^{n} .
$$

Then we have $E_{k} \in M_{k}\left(\Gamma_{0}(1)\right)$, and for primes $p \geq 5$, we have $E_{p-1} \equiv 1(\bmod p)$. We also require Dedekind's $\eta$-function, defined by

$$
\eta(z):=q^{1 / 24} \prod_{n=1}^{\infty}\left(1-q^{n}\right),
$$

and Ramanujan's $\Delta$-function,

$$
\Delta(z):=\eta(z)^{24} \in S_{12}\left(\Gamma_{0}(1)\right) .
$$


Next, we require certain standard operators on modular forms. For positive integers $d$, we define the operators $U_{d}$ and $V_{d}$ on formal power series in $q$ by

$$
\begin{aligned}
\sum_{n=0}^{\infty} a(n) q^{n} \mid U_{d} & :=\sum_{n=0}^{\infty} a(d n) q^{n}, \\
\sum_{n=0}^{\infty} a(n) q^{n} \mid V_{d} & :=\sum_{n=0}^{\infty} a(n) q^{d n} .
\end{aligned}
$$

When acting on spaces of modular forms, we have

$$
V_{d}: M_{k}\left(\Gamma_{0}(N), \chi\right) \rightarrow M_{k}\left(\Gamma_{0}(N d), \chi\right),
$$

and if $d \mid N$, we have

$$
U_{d}: M_{k}\left(\Gamma_{0}(N), \chi\right) \rightarrow M_{k}\left(\Gamma_{0}(N), \chi\right) .
$$

The $U_{d}$ operator has the additional factorization property that

$$
\left[\left(\sum_{n=0}^{\infty} a(n) q^{d n}\right)\left(\sum_{m=0}^{\infty} b(m) q^{m}\right)\right] \mid U_{d}=\left(\sum_{n=0}^{\infty} a(n) q^{n}\right)\left(\sum_{m=0}^{\infty} b(d m) q^{m}\right) .
$$

For positive integers $m$, the Hecke operator $T_{m, k, \chi}$ is an endomorphism on $M_{k}\left(\Gamma_{0}(N), \chi\right)$ and preserves cusp forms. For primes $p \nmid N$ we have

$$
T_{p, k, \chi}:=U_{p}+\chi(p) p^{k-1} V_{p} .
$$

In the setting of modular forms with $p$-integral coefficients, it follows that $T_{p, k, \chi}$ and $U_{p}$ agree modulo $p$.

We also recall the notion of twisting. Let $\varepsilon$ be a Dirichlet character modulo $M$, and let $f(z)=\sum_{n=0}^{\infty} a(n) q^{n}$. Then we define the twist of $f$ by $\varepsilon$ as

$$
f(z) \otimes \varepsilon:=\sum_{n=0}^{\infty} \varepsilon(n) a(n) q^{n} .
$$

If $f(z) \in M_{k}\left(\Gamma_{0}(N), \chi\right)$, let $N^{\prime}$ be the conductor of $\chi$, and define $\mathcal{N}:=\operatorname{lcm}\left(N, N^{\prime} M\right.$, $M^{2}$ ). Then we have $f(z) \otimes \varepsilon \in M_{k}\left(\Gamma_{0}(\mathcal{N}), \varepsilon^{2} \chi\right)$ (see for example AL78).

Next we require basic facts on half-integral weight modular forms. Let $4 \mid N$, and let $\lambda \geq 0$ be an integer. We use $M_{\lambda+\frac{1}{2}}\left(\tilde{\Gamma}_{0}(N), \chi\right)$ to denote the space of holomorphic modular forms of weight $\lambda+\frac{1}{2}$ on $\tilde{\Gamma}_{0}(N)$ with Dirichlet character $\chi$, and we denote by $S_{\lambda+\frac{1}{2}}\left(\tilde{\Gamma}_{0}(N), \chi\right)$ its subspace of cusp forms. For later purposes we note that $\eta(24 z) \in S_{\frac{1}{2}}\left(\tilde{\Gamma}_{0}(576), \chi_{12}\right)$. Let $p$ be prime and let $f(z)=\sum_{n=0}^{\infty} a(n) q^{n} \in$ $M_{\lambda+\frac{1}{2}}\left(\tilde{\Gamma}_{0}(N), \chi\right)$. Then the half-integral weight Hecke operator $T_{p^{2}, \lambda, \chi}$ is defined by

$$
f(z) \mid T_{p^{2}, \lambda+\frac{1}{2}, \chi}:=\sum_{n=0}^{\infty}\left(a\left(p^{2} n\right)+\chi^{*}(p)\left(\frac{n}{p}\right) a(n)+\chi^{*}\left(p^{2}\right) p^{2 \lambda-1} a\left(n / p^{2}\right)\right) q^{n},
$$

where $\chi^{*}(n):=\left(\frac{-1}{n}\right)^{\lambda} \chi(n)$ and $a\left(n / p^{2}\right):=0$ if $p^{2} \nmid n$. As in the integer weight setting, $T_{p^{2}, \lambda+\frac{1}{2}, \chi}$ is an endomorphism on $M_{\lambda+\frac{1}{2}}\left(\tilde{\Gamma}_{0}(N), \chi\right)$ and preserves cusp forms. 
We now state the important theorems of Shimura and Waldspurger. Let $N$ and $\chi$ be as above, and let $\lambda$ and $t \geq 1$ be integers with $t$ square-free. Let $f(z)=$ $\sum_{n=1}^{\infty} a_{f}(n) q^{n} \in S_{\lambda+\frac{1}{2}}\left(\tilde{\Gamma}_{0}(N), \chi\right)$. We define

$$
S_{t}(f)(z):=\sum_{n=1}^{\infty} A_{f, t}(n) q^{n}
$$

by

$$
\sum_{n=1}^{\infty} A_{f, t}(n) n^{-s}=L\left(\chi_{t}^{(\lambda)}, s-\lambda+1\right) \sum_{m=1}^{\infty} a_{f}\left(t m^{2}\right) m^{-s},
$$

where $\chi_{t}^{(\lambda)}(\cdot):=\chi^{*}(\cdot) \chi_{t}(\cdot)$ is a character modulo $N t$.

Theorem 2.1 (Shimura Shi73]). We have

$$
S_{t}(f) \in \begin{cases}M_{2 \lambda}\left(\Gamma_{0}(N / 2), \chi^{2}\right) & \text { if } \lambda=1, \\ S_{2 \lambda}\left(\Gamma_{0}(N / 2), \chi^{2}\right) & \text { if } \lambda>1 .\end{cases}
$$

Moreover, $S_{t}$ commutes with Hecke operators:

$$
S_{t}\left(f \mid T_{p^{2}, \lambda+\frac{1}{2}, \chi}\right)=S_{t}(f) \mid T_{p, 2 \lambda, \chi^{2}} .
$$

An immediate consequence is that if $f(z)$ is a half-integral weight eigenform, then $S_{t}(f)$ will be an integral weight eigenform. For our purposes we set $t=1$ and define $S:=S_{1}$.

We now state Waldspurger's result. Let $\lambda \geq 2$, let $f(z) \in S_{\lambda+\frac{1}{2}}\left(\tilde{\Gamma}_{0}(N), \chi\right)$ be an eigenform for almost all $T_{p^{2}, \lambda+\frac{1}{2}, \chi}$, and let $F(z)=S(f)(z) \in S_{2 \lambda}\left(\Gamma_{0}(N / 2), \chi^{2}\right)$.

Theorem 2.2 (Waldspurger Wal81]). Suppose that $n_{1}, n_{2}$ are positive square-free integers such that for all $p \mid N$, we have $\frac{n_{1}}{n_{2}} \in \mathbb{Q}_{p}^{x^{2}}$. Then we have

$a_{f}\left(n_{1}\right)^{2} L\left(F \otimes\left(\chi^{*}\right)^{-1} \chi_{n_{2}}, \lambda\right) \chi\left(n_{2}\right) n_{2}^{\lambda-\frac{1}{2}}=a_{f}\left(n_{2}\right)^{2} L\left(F \otimes\left(\chi^{*}\right)^{-1} \chi_{n_{1}}, \lambda\right) \chi\left(n_{1}\right) n_{1}^{\lambda-\frac{1}{2}}$.

Remark 2.3. If $\lambda=1$ and $f$ is in the orthogonal complement of the space spanned by single variable theta series, then $S(f)$ is a cusp form, and Theorem 2.2 continues to hold.

\section{Proof of main theorem}

To prove Theorem 1.1 for $\ell \in\{5,7,11\}$, we will identify half-integer weight eigenforms $f_{\ell}$ that are generating functions for $\frac{p\left(\ell n+\delta_{\ell}\right)}{\ell}$. We will then show that $G_{\ell} \otimes \chi_{12}=S\left(f_{\ell}\right)$ (with $G_{\ell}$ as in (1.7)); to conclude we will apply Waldspurger's Theorem.

Lemma 3.1. For $\ell \in\{5,7,11\}$, define

$$
f_{\ell}(z):= \begin{cases}\eta(24 z)^{19} & \ell=5 \\ \eta(24 z)^{17} & \ell=7 \\ \eta(24 z)^{13} E_{8}(24 z) & \ell=11 .\end{cases}
$$

Then we have

$$
\sum_{n \geq 0} \frac{p\left(\ell n+\delta_{\ell}\right)}{\ell} q^{24 n+r_{\ell}} \equiv f_{\ell} \quad(\bmod \ell)
$$


Proof. To prove the congruence we examine $\Delta(z)^{\left(\ell^{2}-1\right) / 24} \mid U_{\ell}$. We first note that the forms $\Delta(z)^{\left(\ell^{2}-1\right) / 24} \mid T_{\ell, \frac{\ell^{2}-1}{2}} \in S_{\frac{\ell^{2}-1}{2}}\left(\Gamma_{0}(1)\right)$ have integer coefficients. For $\ell \in\{5,7,11\}$, one may easily verify that the coefficients of $\Delta(z)^{\left(\ell^{2}-1\right) / 24} \mid T_{\ell, \frac{\ell^{2}-1}{2}}$ of index up to $\left(\ell^{2}-1\right) / 24$ are congruent to zero modulo $\ell$. By a theorem of Sturm (see Ono04, Thm. 2.58), it follows that

$$
\Delta(z)^{\left(\ell^{2}-1\right) / 24} \mid T_{\ell} \equiv 0 \quad(\bmod \ell) .
$$

Hence, the forms $\frac{1}{\ell} \Delta(z)^{\left(\ell^{2}-1\right) / 24} \mid T_{\ell, \frac{\ell^{2}-1}{2}} \in S_{\left(\ell^{2}-1\right) / 2}\left(\Gamma_{0}(1)\right)$ have integer coefficients. Applying Sturm's Theorem again together with the congruence $E_{\ell-1} \equiv 1$ $(\bmod \ell)$, we find that

$$
\frac{1}{\ell} \Delta(z)^{\left(\ell^{2}-1\right) / 24} \mid T_{\ell, \frac{\ell^{2}-1}{2}} \equiv\left\{\begin{array}{lll}
\Delta(z) & (\bmod \ell) & \ell=5, \\
\Delta(z) E_{6}(z)^{2} \equiv \Delta(z) & (\bmod \ell) & \ell=7, \\
\Delta(z) E_{8}(z) E_{10}(z)^{4} \equiv \Delta(z) E_{8}(z) & (\bmod \ell) & \ell=11 .
\end{array}\right.
$$

Since $U_{\ell}$ and $T_{\ell, \frac{\ell^{2}-1}{2}}$ agree on modular forms with integer coefficients modulo $\ell$, we have

$$
\frac{1}{\ell} \Delta(z)^{\left(\ell^{2}-1\right) / 24} \mid U_{\ell} \equiv\left\{\begin{array}{lll}
\Delta(z) & (\bmod \ell) & \ell=5,7, \\
\Delta(z) E_{8}(z) & (\bmod \ell) & \ell=11 .
\end{array}\right.
$$

Using (1.1), (1.2), and (2.1) we see, on the other hand, that

$$
\begin{aligned}
\frac{1}{\ell} \Delta(z)^{\left(\ell^{2}-1\right) / 24} \mid U_{\ell} & =\frac{1}{\ell} q^{\left(\ell^{2}-1\right) / 24} \prod_{n=1}^{\infty} \frac{\left(1-q^{n}\right)^{\ell^{2}}}{\left(1-q^{n}\right)} \mid U_{\ell} \\
& \equiv \prod_{n=1}^{\infty}\left(1-q^{n}\right)^{\ell}\left(\sum_{n=\left(\ell^{2}-1\right) / 24}^{\infty} \frac{p\left(n-\frac{\ell^{2}-1}{24}\right)}{\ell} q^{n}\right) \mid U_{\ell} \\
& \equiv \prod_{n=1}^{\infty}\left(1-q^{n}\right)^{\ell} \sum_{n=\left\lfloor\frac{\ell}{24}\right\rfloor+1}^{\infty} \frac{p\left(\ell n-\frac{\ell^{2}-1}{24}\right)}{\ell} q^{n} \\
& \equiv q^{\left\lfloor\frac{\ell}{24}\right\rfloor+1} \prod_{n=1}^{\infty}\left(1-q^{n}\right)^{\ell} \sum_{n=0}^{\infty} \frac{p\left(\ell n+\delta_{\ell}\right)}{\ell} q^{n} \quad(\bmod \ell) .
\end{aligned}
$$

By comparing (3.1) and (3.2) and solving for $\sum_{n=0}^{\infty} \frac{p\left(\ell n+\delta_{\ell}\right)}{\ell} q^{n}$, we see that

$$
\sum_{n=0}^{\infty} \frac{p\left(\ell n+\delta_{\ell}\right)}{\ell} q^{n} \equiv\left\{\begin{array}{lll}
\prod_{n=1}^{\infty}\left(1-q^{n}\right)^{19} & (\bmod \ell) & \ell=5, \\
\prod_{n=1}^{\infty}\left(1-q^{n}\right)^{17} & (\bmod \ell) & \ell=7, \\
\prod_{n=1}^{\infty}\left(1-q^{n}\right)^{13} E_{8}(z) & (\bmod \ell) & \ell=11 .
\end{array}\right.
$$

By substituting $q^{24}$ for $q$ and multiplying both sides by $q^{r_{\ell}}$, in each case we get our result.

Next, we observe that the forms $f_{\ell}$ are eigenforms for the Hecke operators. 
Lemma 3.2 (Garvan Gar07]). If $\ell \in\{5,7,11\}$, then $f_{\ell} \in S_{\lambda_{\ell}+\frac{1}{2}}\left(\tilde{\Gamma}_{0}(576), \chi_{12}\right)$ is a Hecke eigenform for all Hecke operators $T_{p^{2}, \lambda_{\ell}+\frac{1}{2}, \chi_{12}}$.

This is proved as part of Corollary 3.2 in Gar07. Since $G_{\ell} \in S_{2 \lambda_{\ell}}\left(\Gamma_{0}(6)\right)$ is a newform, it follows by Theorem 3.1 (and its corollary) of [AL78] that $G_{\ell} \otimes \chi_{12} \in$ $S_{2 \lambda_{\ell}}\left(\Gamma_{0}(144)\right)$ is a newform. Moreover, we prove the following:

Lemma 3.3. If $\ell \in\{5,7,11\}$, then we have $G_{\ell} \otimes \chi_{12}=S\left(f_{\ell}\right)$.

Proof. For $\ell \in\{5,7,11\}$, we have $S\left(f_{\ell}\right)$ and $G_{\ell} \otimes \chi_{12} \in S_{2 \lambda_{\ell}}\left(\Gamma_{0}(288)\right)$, and we define $M_{\ell}:=\operatorname{dim} S_{2 \lambda_{\ell}}\left(\Gamma_{0}(288)\right)$. Applying standard formulas (see for example Ono04]), we find that

$$
M_{\ell}= \begin{cases}800 & \ell=5, \\ 704 & \ell=7, \\ 1280 & \ell=11 .\end{cases}
$$

Therefore, to prove the lemma, we calculate the first $M_{\ell}$ coefficients of $S\left(f_{\ell}\right)$ and $G_{\ell} \otimes \chi_{12}$ and verify that they agree. For details of this calculation, see Section 4.

We finalize the proof of Theorem 1.1 by applying Theorem 2.2 with $f=f_{\ell}, F=$ $G_{\ell} \otimes \chi_{12}, n_{1}=r_{\ell}$ and $n_{2}=24 n+r_{\ell}$. Since $n_{1} \equiv n_{2}(\bmod 24)$, we have $\frac{n_{2}}{n_{1}} \in \mathbb{Q}_{p}^{\times^{2}}$ for $p \in\{2,3\}$. We calculate that $\chi_{12}^{*}(\cdot)\left(\frac{24 n+r_{\ell}}{\cdot}\right)=\left(\frac{(-1)^{\lambda} \ell\left(24 n+r_{\ell}\right)}{\cdot}\right)=\chi_{D(\ell, n)}(\cdot)$. Thus, we have

$$
\frac{L\left(G_{\ell} \otimes \chi_{D(\ell, n)}, \lambda_{\ell}\right)\left(24 n+r_{\ell}\right)^{\lambda_{\ell}-\frac{1}{2}}}{L\left(G_{\ell} \otimes \chi_{D(\ell, 0)}, \lambda_{\ell}\right)\left(r_{\ell}\right)^{\lambda_{\ell}-\frac{1}{2}}}=\frac{a_{f_{\ell}}\left(24 n+r_{\ell}\right)^{2}}{a_{f_{\ell}}\left(r_{\ell}\right)^{2}} .
$$

Noting that $a_{f_{\ell}}\left(24 n+r_{\ell}\right) \equiv \frac{p\left(\ell n+\delta_{\ell}\right)}{\ell}$ modulo $\ell$ and $a_{f_{\ell}}\left(r_{\ell}\right)=1$, the proof is complete.

\section{Appendix}

To compare $S\left(f_{\ell}\right)$ and $G_{\ell} \otimes \chi_{12}$, we will need to compute the first $M_{\ell}$ coefficients for each form. Set $p_{\ell}$ to be the largest prime less than $M_{\ell}$. Then to compute $S\left(f_{\ell}\right)$ we must compute the first $p_{\ell}^{2} r_{\ell}$ coefficients of $f_{\ell}$. To compute these coefficients we employ well-known formulas for $\eta(24 z)$ and $\eta(24 z)^{3}$ :

$$
\begin{gathered}
\eta(24 z)=1+\sum_{n \geq 1}(-1)^{n}\left(q^{12 n(3 n-1)}+q^{12 n(3 n+1)}\right), \\
\eta(24 z)^{3}=\sum_{n \geq 0}(-1)^{n}(2 n+1) q^{3(2 n+1)^{2}} .
\end{gathered}
$$

The first formula is Euler's Pentagonal Number Theorem, and the second follows from Jacobi's Triple Product Identity. These formulas allow us to rapidly calculate the necessary number of coefficients for $\eta(24 z)$ and $\eta(24 z)^{3}$ on a computer, which we use to build $\eta^{13}(24 z), \eta^{17}(24 z)$, and $\eta^{19}(24 z)$.

For the most efficient use of computer memory available to us, the powers of $\eta$ were encoded as arrays and then saved as text files. To multiply them together, we wrote simple programs which kept our memory use under 2 GB of RAM. The text file for the coefficients of $\eta^{19}(24 z)$ was approximately $82 \mathrm{MB}$.

The calculation of the necessary coefficients for $S\left(f_{5}\right)$ and $S\left(f_{7}\right)$ is straightforward from the definition. However, for $f_{11}=\eta^{13}(24 z) E_{8}(24 z)$, the Eisenstein factor makes the size of the coefficients significantly larger. Therefore, to conserve file space, we employ a Chinese Remainder Theorem argument. 
For the integer weight newforms $G_{\ell}$, we follow a similar argument as in GO99]. We identify $G_{\ell}^{*} \in S_{2 \lambda_{\ell}}\left(\Gamma_{0}(6)\right)$, which are linear combinations of $\eta$-products, such that $G_{\ell} \otimes \chi_{1}=G_{\ell}^{*} \otimes \chi_{1}$ in $S_{2 \lambda_{\ell}}\left(\Gamma_{0}(36)\right)$, where $\chi_{1}$ denotes the trivial character modulo 6. Hence, we have $G_{\ell} \otimes \chi_{12}=G_{\ell}^{*} \otimes \chi_{12}$. From the bound for the dimension of $S_{2 \lambda_{\ell}}\left(\Gamma_{0}(36)\right)$ coming from the valence formula, we prove this equality of modular forms by checking that the first $12 \lambda_{\ell}$ coefficients of the forms agree. One uses standard techniques to compute the required number of coefficients of the newform $G_{\ell}$, and the forms $G_{\ell}^{*}$ are given explicitly as follows:

$$
\begin{aligned}
& G_{5}^{*}:=-1140 \eta(z)^{30} \eta(3 z)^{6}-40643208 \eta(z)^{6} \eta(3 z)^{6} \eta(6 z)^{24} \\
& -3147616 \eta(z) \eta(2 z)^{25} \eta(3 z)^{5} \eta(6 z)^{5}+\eta(z)^{29} \eta(2 z)^{5} \eta(3 z) \eta(6 z) \mid U_{2} \\
& +36120492 \eta(2 z)^{6} \eta(3 z)^{24} \eta(6 z)^{6}-42133284 \eta(z)^{10} \eta(2 z)^{4} \eta(3 z)^{2} \eta(6 z)^{20} \\
& +2184396 \eta(z)^{10} \eta(2 z)^{16} \eta(3 z)^{2} \eta(6 z)^{8}-71788 \eta(z)^{25} \eta(2 z) \eta(3 z)^{5} \eta(6 z)^{5}, \\
& G_{7}^{*}:=-4110102 \eta(z)^{2} \eta(2 z)^{2} \eta(3 z)^{2} \eta(6 z)^{26}-1239300 \eta(z)^{2} \eta(2 z)^{14} \eta(3 z)^{2} \eta(6 z)^{14} \\
& +1166400 \eta(z)^{3} \eta(2 z)^{9} \eta(3 z)^{7} \eta(6 z)^{13}-729 \eta(z)^{2} \eta(2 z)^{2} \eta(3 z)^{26} \eta(6 z)^{2} \\
& -13414 \eta(z)^{2} \eta(2 z)^{26} \eta(3 z)^{2} \eta(6 z)^{2}+87 \eta(z)^{26} \eta(2 z)^{2} \eta(3 z)^{2} \eta(6 z)^{2} \\
& +\eta(z)^{26} \eta(2 z)^{2} \eta(3 z)^{2} \eta(6 z)^{2} \mid U_{2} \text {, } \\
& G_{11}^{*}:=\eta(z)^{50} \eta(2 z)^{2} \eta(3 z)^{2} \eta(6 z)^{2} \mid U_{3} \\
& +\frac{6104578068776124145}{751160222919} \eta(z)^{50} \eta(2 z)^{2} \eta(3 z)^{2} \eta(6 z)^{2} \\
& +\frac{13103131110144431}{8012375711136} \eta(z)^{45} \eta(2 z)^{3} \eta(3 z) \eta(6 z)^{7} \mid U_{2} \\
& -\frac{3299973272427100336}{751160222919} \eta(z)^{40} \eta(2 z)^{16} \\
& +\frac{309477427592855}{27820748997} \eta(z)^{38} \eta(2 z)^{2} \eta(3 z)^{14} \eta(6 z)^{2} \mid U_{2} \\
& +\frac{17128468858079251316}{751160222919} \eta(z)^{37} \eta(2 z)^{7} \eta(3 z)^{9} \eta(6 z)^{3} \\
& -\frac{162517745107473935}{8012375711136} \eta(z)^{37} \eta(2 z)^{7} \eta(3 z)^{9} \eta(6 z)^{3} \mid U_{2} \\
& -\frac{2550874792797073672288}{83462246991} \eta(z)^{36} \eta(3 z)^{4} \eta(6 z)^{16} \\
& -\frac{561555892954984790068}{83462246991} \eta(z)^{33} \eta(2 z)^{3} \eta(3 z)^{13} \eta(6 z)^{7} \\
& +\frac{3540256483941586597376}{751160222919} \eta(z)^{31} \eta(2 z)^{13} \eta(3 z)^{3} \eta(6 z)^{9} \\
& -\frac{8342412746725559808}{38162893} \eta(z)^{31} \eta(2 z) \eta(3 z)^{3} \eta(6 z)^{21} \\
& +\frac{201341803552556544}{38162893} \eta(z)^{24} \eta(3 z)^{16} \eta(6 z)^{16} \\
& +\frac{238873453748975747072}{751160222919} \eta(z)^{16} \eta(2 z)^{40} \text {. }
\end{aligned}
$$

Finally we verify that $G_{\ell}^{*} \otimes \chi_{12}=S\left(f_{\ell}\right)$. For $\ell=11$ we need bounds on the size of the coefficients in order to employ the Chinese Remainder Theorem. Based on 
Deligne's proof of the Weil Conjectures, we have the following bound for the size of the coefficients of newforms.

If $f(z)=\sum_{n=1}^{\infty} a_{f}(n) q^{n} \in S_{k}\left(\Gamma_{0}(N)\right)$ is a newform, then

$$
\left|a_{f}(n)\right| \leq \sum_{1 \leq d \mid n} n^{(k-1) / 2}
$$

This implies that $\left|a_{S\left(f_{11}\right)}(n)\right|,\left|a_{G_{11}}(n)\right|<10^{44}$ for $n \leq 1280$. Finally, we finish the proof of Lemma 3 by verifying that the first 1,280 coefficients of $G_{11}^{*} \otimes \chi_{12}$ and $S\left(f_{11}\right)$ agree modulo $p$, where $p$ ranges over the first 8 primes greater than $10^{6}$.

\section{REFERENCES}

[Ahl00] S. Ahlgren, Distribution of the partition function modulo composite integers $M$. Math. Ann. 318 (2000), no. 4, 795-803. MR1802511 (2001j:11099)

[AB03] S. Ahlgren and M. Boylan, Arithmetic properties of the partition function. Invent. Math. 153 (2003), no. 3, 487-502. MR2000466 (2004e:11115)

[AO01] S. Ahlgren and K. Ono, Congruence properties for the partition function. Proc. Natl. Acad. Sci. USA 98 (2001), no. 23, 12882-12884. MR.1862931 (2002k:11187)

[And98] G. E. Andrews, The Theory of Partitions. Cambridge University Press, Cambridge, 1998. MR.1634067 (99c:11126)

[AL78] A.O.L. Atkin and W.C.W. Li, Twists of newforms and pseudo-eigenvalues of $W$ operators. Invent. Math. 48 (1978), no. 3, 221-243. MR508986 (80a:10040)

[Gar07] F.G. Garvan, Congruences for Andrews' smallest parts partition function and new congruences for Dyson's rank. Int. J. Number Theory, to appear. arXiv:0710.5793

[GO99] L. Guo and K. Ono, The partition function and the arithmetic of certain modular L-functions. Internat. Math. Res. Notices 1999, no. 21, 1179-1197. MR1728677 (2000m:11102)

[Iwa97] H. Iwaniec, Topics in Classical Automorphic Forms. Graduate Studies in Mathematics, 17. American Mathematical Society, Providence, RI, 1997. MR1474964 (98e:11051)

[Kno93] M. Knopp, Modular Functions in Analytic Number Theory. Chelsea Publishing Company, New York, 1993. MR0265287 (42:198)

[Ono00] K. Ono, Distribution of the partition function modulo m. Ann. of Math. (2) 151 (2000), no. 1, 293-307. MR 1745012 (2000k:11115)

[Ono04] K. Ono, The Web of Modularity: Arithmetic of the Coefficients of Modular Forms and $q$-Series. CBMS Regional Conference Series in Mathematics, 102. American Mathematical Society, Providence, RI, 2004. MR2020489 (2005c:11053)

[Ono08] K. Ono, Unearthing the Visions of a Master: Harmonic Maass Forms and Number Theory. Harvard-MIT Current Developments in Mathematics 2008, International Press, (preliminary version).

[Shi73] G. Shimura, On modular forms of half integral weight. Ann. of Math. (2) 97 (1973), 440-481. MR0332663 (48:10989)

[Wal81] J.-L. Waldspurger, Sur les coefficients de Fourier des formes modulaires de poids demientier. J. Math. Pures Appl. (9) 60 (1981), no. 4, 375-484. MR646366 (83h:10061)

Department of Mathematics, University of South Carolina, Columbia, South CaroLINA 29208

E-mail address: webbjj3@mailbox.sc.edu 